\title{
On tree-decompositions of one-ended graphs
}

\author{
Johannes Carmesin*, Florian Lehner ${ }^{\dagger}$, and Rögnvaldur G. Möller ${ }^{\ddagger}$
}

November 5, 2018

\begin{abstract}
A graph is one-ended if it contains a ray (a one way infinite path) and whenever we remove a finite number of vertices from the graph then what remains has only one component which contains rays. A vertex $v$ dominates a ray in the end if there are infinitely many paths connecting $v$ to the ray such that any two of these paths have only the vertex $v$ in common. We prove that if a one-ended graph contains no ray which is dominated by a vertex and no infinite family of pairwise disjoint rays, then it has a tree-decomposition such that the decomposition tree is one-ended and the tree-decomposition is invariant under the group of automorphisms.

This can be applied to prove a conjecture of Halin from 2000 that the automorphism group of such a graph cannot be countably infinite and solves a recent problem of Boutin and Imrich. Furthermore, it implies that every transitive one-ended graph contains an infinite family of pairwise disjoint rays.
\end{abstract}

\section{Introduction}

The ends of a graph $G$ are defined as equivalence classes of rays (one sided infinite paths). Two rays are said to belong to the same end if for every finite set $F$ of vertices the same component of $G \backslash F$ contains infinitely many vertices from both rays. The ends of a graph are a tool to capture the "large-scale" structure of an infinite graphs. In particular if a graph has more than one end then the graph can be said to be "tree-like". In 9], Dunwoody and Krön constructed so called structure trees to describe this "tree-likeness" of a graph with more than one end. A structure tree is constructed from a nested Aut $(G)$ invariant family of separations of the graph such that the action of Aut $(G)$ on the family of separations gives an action of $\operatorname{Aut}(G)$ on the tree. The work of Dunwoody and Krön builds on the book 5 by Dicks and Dunwoody, see also a recent account of this theory in 8 .

Tree-decompositions (defined in Section 3) are very similar to structure trees; and do play a central role in Graph Minor Theory and are a standard tool in

\footnotetext{
*Department of Pure Mathematics and Mathematical Statistics, University of Cambridge Wilberforce Road, Cambridge CB3 0WB, United Kingdom

${ }^{\dagger}$ Mathematics Institute, University of Warwick, Zeeman Building, Coventry CV4 7AL, United Kingdom

Florian Lehner was supported by the Austrian Science Fund (FWF), grant J 3850-N32

${ }^{\ddagger}$ Science Institute, University of Iceland, IS-107 Reykjavík, Iceland

Rögnvaldur G. Möller acknowledges support from the University of Iceland Research Fund
} 


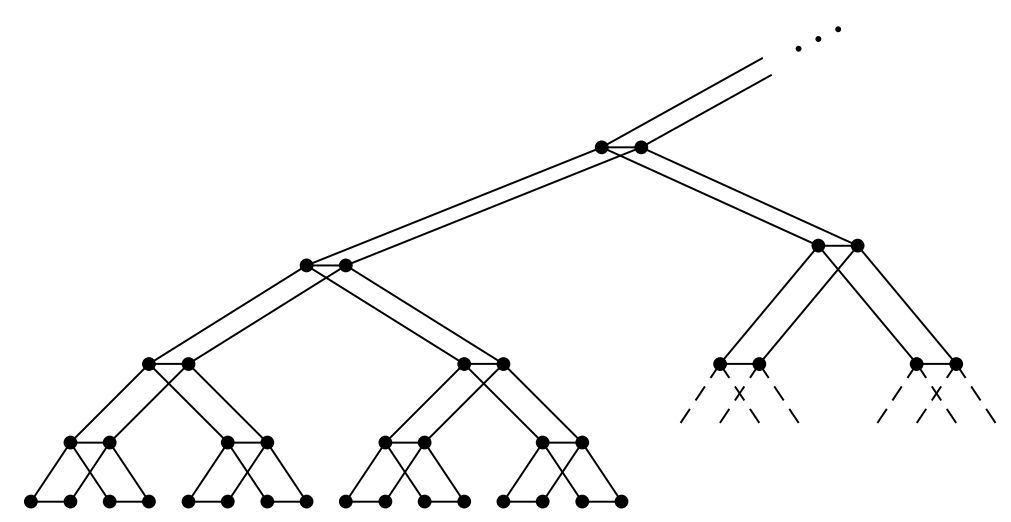

Figure 1: The product of the canopy tree with $K_{1}$. This graph has a tree decomposition whose decomposition tree is the canopy tree.

Graph Theory to describe tree-structure [6]. If the nested set of separations of a tree-decomposition is $\operatorname{Aut}(G)$-invariant then the tree-decomposition is also Aut $(G)$-invariant and $\operatorname{Aut}(G)$ acts on the decomposition tree.

Dunwoody and Krön apply their construction to obtain a combinatorial proof of generalization of Stalling's theorem of groups with at least two ends. This method has multifarious other applications, as demonstrated by Hamann in [18] and Hamann and Hundertmark in [19].

However, for graphs with only a single end, such as the 2-dimensional grid, these structure trees and the related tree-decompositions may be trivial. Hence such a structural understanding of this class of graphs remains elusive. If the end of a one-ended graph has finite vertex degree, that is, there is no infinite set of pairwise vertex-disjoint rays belonging to that end, then Halin showed in 1965 [12 that there are tree-decompositions displaying the end. A precise definition can be found towards the end of Section 3 , but essentially this means that the tree also only has one end and that end "corresponds" to the end of the graph is a precise way. Nevertheless, for these tree-decompositions to be of any use for applications as above, one needs them to have the additional property that they are invariant under the group of automorphisms. Unfortunately such tree-decompositions do not exist for all graphs in question, see Example 3.10 below. Note that in this example there is a vertex $v$ dominating the end, that is, for every ray in the end there are infinitely many paths connecting $v$ to the ray such that any two of these paths have only the vertex $v$ in common. In this paper we construct such tree-decompositions if the end is not dominated.

Theorem 1.1. Every one-ended graph whose end is undominated and has finite vertex degree has a tree-decomposition that displays its end and that is invariant under the group of automorphisms.

A very simple example is shown in Figure 1

This better structural understanding leads to applications similar to those for graphs with more than one end. Indeed, below we deduce from Theorem 1.1 a conjecture of Halin from 2000, and answer a recent question of Boutin and Imrich. A further application was pointed out by Hamann. 
Applications. In 17 Halin showed that one-ended graphs with vertex degree equal to one cannot have countably infinite automorphism group. Not completely satisfied with his result, he conjectured that this extends to oneended graphs with finite vertex degree. Theorem 1.1 implies this conjecture.

Theorem 1.2. Given a graph with one end which has finite vertex degree, its automorphism group is either finite or has at least $2^{\aleph_{0}}$ many elements.

Theorem 1.2 can be further applied to answer a question posed by Boutin and Imrich, who asked in 1 whether there is a locally finite graph with only one end and linear growth and countably infinite automorphism group. Theorem 1.2 implies a negative answer to this question as well as strengthenings of further results of Boutin and Imrich, see Section 4 for details.

Finally, Matthias Hamann 11 pointed out the following consequence of Theorem 1.1

Theorem 1.3. The end of a transitive one-ended graph must have infinite vertex degree.

This extends a result of Thomassen for locally finite graphs, see 23, Proposition 5.6]. We actually prove a stronger version of Theorem 1.3, see Theorem 5.1, with 'quasi-transitive2 2 in place of 'transitive'.

The rest of this paper is structured as follows: in Section 2 we set up all necessary notations and definitions. As explained in 4, there is a close relation between tree-decompositions and nested sets of separations. In this paper we work mainly with nested sets of separations. In Section 3 we prove Theorems 3.8 and 3.9 that imply Theorem 1.1, and Section 4 is devoted to the proof of Theorem 1.2 and its implications on the work of Boutin and Imrich. Finally, in Section 5 we prove Theorem 5.1 that implies Theorem 1.3

Many of the lemmas we apply in this work were first proved by Halin. Since in some cases we need slight variants of the original results and also since Halin's original papers might not be easily accessible, proofs of some of these results are included in appendices.

\section{Preliminarlies}

Throughout this paper $V(G)$ and $E(G)$ denote the sets of vertices and edges of a graph $G$, respectively. We refer to [6] for all graph theoretic notions which are not explicitly defined.

\subsection{Separations, rays and ends}

A separator in a graph $G$ is a subset $S \subseteq V(G)$ such that $G-S$ is not connected. We say that a separator $S$ separates vertices $u$ and $v$ if $u$ and $v$ are in different components of $G-S$. Given two vertices $u$ and $v$, a separator $S$ separates $u$ and $v$ minimally if it separates $u$ and $v$ and the components of $G-S$ containing

\footnotetext{
${ }^{1}$ personal communication

${ }^{2}$ Here a graph is quasi-transitive, if there are only finitely many orbits of vertices under the automorphism group.
} 
$u$ and $v$ both have the whole of $S$ in their neighbourhood. The following lemma can be found in Halin's 1965 paper [14, Statement 2.4], and also in his later paper [15, Corollary 1] and then with a different proof.

Lemma 2.1. Given vertices $u$ and $v$ and $k \in \mathbb{N}$, there are only finitely many distinct separators of size at most $k$ separating $u$ and $v$ minimally.

A separation is a pair $(A, B)$ of subsets of $V(G)$ such that $A \cup B=V(G)$ and there is no edge connecting $A \backslash B$ to $B \backslash A$. This immediately implies that if $u$ and $v$ are adjacent vertices in $G$ then $u$ and $v$ are both contained in either $A$ or $B$. The sets $A$ and $B$ are called the sides of the separation $(A, B)$. A separation $(A, B)$ is said to be proper if both $A \backslash B$ to $B \backslash A$ are non-empty and then $A \cap B$ is a separator. A separation $(A, B)$ is tight if every vertex in $A \cap B$ has neighbours in both $A \backslash B$ and $B \backslash A$. The order of a separation is the number of vertices in $A \cap B$. Throughout this paper we will only consider separations of finite order. The following is well-known.

Lemma 2.2. (See 3, Lemma 2.1]) Given any two separations $(A, B)$ and $(C, D)$ of $G$ then the sum of the orders of the separations $(A \cap C, B \cup D)$ and $(B \cap D, A \cup$ $C)$ is equal to the sum of the orders of the separations $(A, B)$ and $(C, D)$. In particular if the orders of $(A, B)$ and $(C, D)$ are both equal to $k$ then the sum of the orders of $(A \cap C, C \cup D)$ and $(B \cap D, A \cup C)$ is equal to $2 k$.

The separations $(A, B)$ and $(C, D)$ are strongly nested if $A \subseteq C$ and $D \subseteq B$. They are nested if they are strongly nested after possibly exchanging ' $(A, B)$ ' by ' $(B, A)$ ' or ' $(C, D)$ ' by ' $(D, C)$ '. That is, $(A, B)$ and $(C, D)$ are nested if one of the following holds:

- $A \subseteq C$ and $D \subseteq B$,

- $A \subseteq D$ and $C \subseteq B$,

- $B \subseteq C$ and $D \subseteq A$,

- $B \subseteq D$ and $C \subseteq A$.

We say a set $\mathcal{S}$ of separations is nested, if any two separations in it are nested.

A ray in a graph $G$ is a one-sided infinite path $v_{0}, v_{1}, \ldots$ in $G$. The sub-rays of a ray are called its tails. Given a finite separator $S$ of $G$, there is for every ray $\gamma$ a unique component of $G-S$ that contains all but finitely many vertices of $\gamma$. We say that $\gamma$ lies in that component of $G-S$. Given a separation $(A, B)$ of finite order one can similarly say that $\gamma$ lies in one of the sides of the separation. Two rays are in the same end if they lie in the same component of $G-S$ for every finite separator of $G$. Clearly, this is an equivalence relation. An equivalence class is called a (vertex) end 3 . An alternative way to define ends is to say that two rays $R_{1}$ and $R_{2}$ are in the same end if there are infinitely many pairwise disjoint $R_{1}-R_{2}$ paths. (Given subsets $X$ and $Y$ of the vertex set, an $X-Y$ path is a path that has its initial vertex in $X$ and terminal vertex in $Y$ and every other vertex is neither in $X$ nor $Y$. In the case where $X=\{x\}$ then we speak of $x-Y$ paths instead of $X-Y$ paths and if $Y=\{y\}$ we speak of

\footnotetext{
${ }^{3}$ A notion related to 'vertex ends' are 'topological ends'. In this paper we are mostly interested in graphs where no vertex dominates a vertex end. In this context the two notions of end agree.
} 
$x-y$ paths.) An end $\omega$ lies in a component $C$ of $G-S$ if every ray that belongs to $\omega$ lies in $C$. Clearly, every end lies in a unique component of $G-S$ for every finite separator $S$ and if $(A, B)$ is a separation of finite order then an end either lies in $A$ or $B$.

A vertex $v \in V(G)$ dominates an end $\omega$ of $G$, if there is no separation $(A, B)$ of finite order such that $v \in A \backslash B$ and $\omega$ lies in $B$. Equivalently, $v$ dominates $\omega$ if for every ray $R$ in $\omega$ there are infinitely many paths connecting $v$ to $R$ such that any two of them only intersect in $v$.

The vertex degree of an end $\omega$ is equal to a natural number $k$ if the maximal cardinality of a family of pairwise disjoint rays belonging to the end is $k$. If no such number $k$ exists then we say that the vertex-degree of the end is infinite. Halin [12 (see also [6, Theorem 8.2.5]) proved that if the vertex-degree of an end is infinite then there is an infinite family of pairwise disjoint rays belonging to the end. Ends with finite vertex degree are sometimes called thin and those with infinite vertex degree are called thick.

The following lemma is well-known. A proof can be found in Appendix A.

Lemma 2.3. (Cf. 17, Section 3]) Let $G$ be a connected graph and $\omega$ an end of $G$ having a finite vertex degree. Then there are only finitely many vertices in $G$ that dominate the end $\omega$.

In this paper we are focusing on 1-ended graphs where the end $\omega$ has vertex degree $k$. In the following definition we pick out a class of separations that are relevant in this case.

Definition 2.4. Let $G$ be an arbitrary graph. If $\omega$ is an end of $G$ that has vertex degree $k$ then say that a separation $(A, B)$ is $\omega$-relevant if it has the following properties

- the order of $(A, B)$ is exactly $k$,

- $A \backslash B$ is connected,

- every vertex in $A \cap B$ has a neighbour in $A \backslash B$,

- $\omega$ lies in $B$, and

- there is no separation $(C, D)$ of order $<k$ such that $A \subseteq C$ and $\omega$ lies in $D$.

Define $\mathcal{S}_{\omega}$ as the set of all $\omega$-relevant separations.

The following characterization of $\omega$-relevant separations is a Menger type result. A proof based on $[10$ and $[12$ is contained in Appendix A.

Lemma 2.5. Let $G$ be an arbitrary graph. Suppose $\omega$ is an end of $G$ with vertex degree $k$.

1. If $(A, B)$ is an $\omega$-relevant separation then there is a family of $k$ pairwise disjoint rays in $\omega$ such that each of them has its initial vertex in $A \cap B$.

2. Conversely, if $(A, B)$ is a separation of order $k$ such that $A \backslash B$ is connected, every vertex in $A \cap B$ has a neighbour in $A \backslash B$, the end $\omega$ lies in $B$ and there is a family of $k$ disjoint rays in $\omega$ such that each of these rays has its initial vertex in $A \cap B$ then the separation $(A, B)$ is $\omega$-relevant. 
In particular, for $(A, B) \in \mathcal{S}_{\omega}$ the component of $G-(A \cap B)$ in which $\omega$ lies has the whole of $A \cap B$ in its neighbourhood and hence every separation in $\mathcal{S}_{\omega}$ is tight. Note that the set $A \backslash B$ completely determines the $\omega$-relevant separation $(A, B)$.

The relation

$$
(A, B) \leq(C, D): \Longleftrightarrow A \subseteq C \text { and } B \supseteq D
$$

defines a partial order on the set of all separations, so in particular on the set $\mathcal{S}_{\omega}$. Since $(C, D)$ is a tight separation, the condition $A \subseteq C$ implies that $D \subseteq B$. This is shown in [4, (7) on p. 17] and the argument goes as follows: Suppose that $D \nsubseteq \subset B$ and $x \in D \backslash B$. Then $x \in A \subseteq C$ so $x \in(C \cap D) \backslash B$. Because $(C, D)$ is a tight separation, $x$ has a neighbour $y \in D \backslash C$. But $x \in A \backslash B$ and hence $y$ must also be in $A$. But $y \notin C$, contradicting the assumption that $A \subseteq C$. Hence $D \subseteq B$ and $(A, B) \leq(C, D) \Longleftrightarrow A \subseteq C$.

The next result follow from results of Halin in [12. These results are in turn proved by using Menger's Theorem. For the convenience of the reader a detailed proof is provided in Appendix A.

Theorem 2.6. Let $G$ be a connected 1-ended graph such that the end $\omega$ is undominated and has finite vertex degree $k$. Then there is a sequence $\left\{\left(A_{n}, B_{n}\right)\right\}_{n \geq 0}$ of $\omega$-relevant separations, such that the sequence of sets $B_{n}$ is strictly decreasing and for every finite set of vertices $F$ there is a number $n$ such that $F \subseteq A_{n} \backslash B_{n}$.

We will not use the following in our proof.

Remark 2.7. Theorem [2.6] is also true if we leave out the assumption that $G$ is one-ended (and replace 'the end $\omega$ ' by 'there exists an end $\omega$ that').

\subsection{Automorphism groups}

An automorphism of a graph $G=(V, E)$ is a bijective function $\gamma: V \rightarrow V$ that preserves adjacency and whose inverse also preserves adjacency. Clearly an automorphism $\gamma$ also induces a bijection $E \rightarrow E$ which by abuse of notation we will also call $\gamma$. The automorphism group of $G$, i.e. the group of all automorphisms of $G$, will be denoted by $\operatorname{Aut}(G)$.

Let $\Gamma$ be a subgroup of $\operatorname{Aut}(G)$. For a set $D \subseteq V(G)$ we define the setwise stabiliser of $D$ as the subgroup $\Gamma_{\{D\}}=\{\gamma \in \Gamma \mid \gamma(D)=D\}$ and the pointwise stabiliser of $D$ is defined as $\Gamma_{(D)}=\{\gamma \in \Gamma \mid \gamma(d)=d$ for all $d \in D\}$. The setwise stabiliser is the subgroup of all elements in $\Gamma$ that leave the set $D$ invariant and the pointwise stabiliser is the subgroup of all those elements in $\Gamma$ that fix every vertex in $D$. If $D \subseteq V(G)$ is invariant under $\Gamma$ then we use $\Gamma^{D}$ to denote the permutation group on $D$ induced by $\Gamma$, i.e. $\Gamma^{D}$ is the group of all permutation $\sigma$ of $D$ such that there is some element $\gamma \in \Gamma$ such that the restriction of $\gamma$ to $D$ is equal to $\sigma$. Note that $\Gamma_{(D)}$ is a normal subgroup of $\Gamma_{\{D\}}$ and the index $\Gamma_{(D)}$ in $\Gamma_{\{D\}}$ is equal to the number of elements in $\left(\Gamma_{\{D\}}\right)^{D}$.

The full automorphism group of a graph has a special property relating to separations. Suppose $\gamma$ is an automorphism of a graph $G$ and that $\gamma$ leaves both sides of a separation $(A, B)$ invariant and fixes every vertex in the separator $A \cap B$. Then the full automorphism group contains automorphisms $\sigma_{A}$ and $\sigma_{B}$ such that $\sigma_{A}$ like $\gamma$ on $A$ fixes every vertex in $B$ and vice versa for $\sigma_{B}$. 
Informally one can describe this property by saying that the pointwise stabiliser (in the full automorphism group) of a set $D$ of vertices acts indpendently on the components of $G-D$. We will refer to this property as the independence property.

There is a natural topology on $\operatorname{Aut}(G)$, called the permutation topology: endow the vertex set with the discrete topology and consider the topology of pointwise convergence on $\operatorname{Aut}(G)$. Clearly, the permutation topology also makes sense for any group of permutations of a set. The following lemma is a special case of a result in [2, (2.6) on p. 28]. In particular it tells us that the limit of a sequence of automorphisms again is an automorphism. This fact will be central to the proof of Theorem 1.2

Lemma 2.8. The automorphism group of a graph is closed in the set of all permutations of the vertex set endowed with the topology of pointwise convergence.

The next result is also a special case of a result from Cameron's book refered to above. This time we look at [2, (2.2) on p. 28].

Lemma 2.9. The automorphism group of a countable graph is finite, countably infinite or has at least $2^{\aleph_{0}}$ elements.

\section{Invariant nested sets}

In this section we will prove Theorems 3.8 and 3.9. Theorem 1.1 follows from Theorem 3.9. The following two facts about sequences of nested separations will be useful at several points in the proofs.

Lemma 3.1. Let $G$ be a connected graph. Assume that $\left(A_{i}, B_{i}\right)_{i \in \mathbb{N}}$ is a sequence of proper separations of order at most some fixed natural number $k$. Assume also that $A_{i} \subsetneq A_{i-1}$, every $A_{i} \backslash B_{i}$ is connected, and every vertex in $A_{i} \cap B_{i}$ has a neighbour in $A_{i} \backslash B_{i}$. Define $X$ as the set of vertices contained in infinitely many $A_{i}$. Then

1. $X \subseteq B_{i}$ for all but finitely many $i$,

2. there is a unique end $\mu$ which lies in every $A_{i}$, and

3. $x \in X$ if and only if $x$ dominates $\mu$.

Proof. First observe that $X=\bigcap_{i \in \mathbb{N}} A_{i}$ because the sequence $A_{i}$ is decreasing. Let $X^{\prime}$ be the set of vertices in $X$ with a neighbour outside of $X$. For every $x \in X^{\prime}$ we can find a neighbour $y$ of $x$ and $i_{0} \in \mathbb{N}$ such that $y \notin A_{i}$ for every $i \geq i_{0}$. Since the edge $x y$ must be contained in either $A_{i}$ or $B_{i}$ we conclude that $x \in B_{i}$ and thus $x \in A_{i} \cap B_{i}$ for $i \geq i_{0}$.

Hence there is $i_{1} \in \mathbb{N}$ such that $X^{\prime} \subseteq A_{i} \cap B_{i}$ for every $i \geq i_{1}$. The order of each separation is at most $k$, so $X^{\prime}$ contains at most $k$ vertices. Now for $i \geq i_{1}$ every path from $X \backslash B_{i}$ to $A_{i} \backslash\left(X \cup B_{i}\right)$ must pass through $X^{\prime}$ and thus through $B_{i}$. Since $A_{i} \backslash B_{i}$ is connected this means that one of the two sets must be empty, i.e., either $X \backslash B_{i}=\emptyset$ or $X \backslash B_{i}=A_{i} \backslash B_{i}$. Assume that the latter is the case. Then $A_{i}$ contains at most $k$ vertices which are not contained in $X$ and the same is clearly true for every $A_{j}$ for $j>i$. This contradicts the fact that the sequence $A_{i}$ was assumed to be infinite and strictly decreasing. We conclude 
that $X \subseteq B_{i}$ for $i \geq i_{1}$. Note that this implies that $X=X^{\prime}$ because if $i \geq i_{1}$ then $X \subseteq A_{i} \cap B_{i}$ and every vertex in $A_{i} \cap B_{i}$ has an neighbour in $A_{i} \backslash B_{i}$.

To see that there is an end $\mu$ which lies in every $A_{i}$ we construct a ray which has a tail in each $A_{i}$. For this purpose pick for $i \geq i_{1}$ a vertex $v_{i} \in A_{i} \backslash X$ and paths $P_{i}$ connecting $v_{i}$ to $v_{i+1}$ in $A_{i} \backslash X$. This is possible because $A_{i} \backslash X$ contains $A_{i} \backslash B_{i}$ and is connected $\left(A_{i} \backslash B_{i}\right.$ is connected and every vertex in $B_{i} \cap A_{i}$ has a neighbour in $A_{i} \backslash B_{i}$ ). No vertex lies on infinitely many paths $P_{i}$ because no vertex is contained in infinitely many sets $A_{i} \backslash X$. Hence the union of the paths $P_{i}$ is an infinite, locally finite graph and thus contains a ray. This ray belongs to an end $\mu$ which lies in every $A_{i}$.

Finally we need to show that every vertex in $X$ dominates the end $\mu$. Without loss of generality we can assume that $X \subseteq B_{i}$ for all $i$. So, let $R$ be a ray in $\mu$ and $x \in X$. We will inductively construct infinitely many paths from $x$ to $R$ which only intersect in $x$. Assume that we already constructed some finite number of such paths. Since all of them have finite length, there is an index $i$ such that $A_{i} \backslash B_{i}$ doesn't contain any vertex in their union. The ray $R$ has a tail contained in $A_{i} \backslash B_{i}$ and since $x \in A_{i} \cap B_{i}$ we know that $x$ has a neighbour in $A_{i} \backslash B_{i}$. Finally $A_{i} \backslash B_{i}$ is connected, so we can find a path connecting $x$ to the tail of $R$ which intersects the previously constructed paths only in $x$. Proceeding inductively we obtain infinitely many paths connecting $x$ to $R$ which pairwise only intersect in $x$ completing the proof of the Lemma.

We would now like to construct a subset of the set $\mathcal{S}_{\omega}$ of $\omega$-relevant separations that is both nested and invariant under all automorphisms and from that set we construct a tree. The following two lemmas give us important properties of nestedness when we restrict to $\omega$-relevant separations.

Lemma 3.2. Two separations $(A, B),(C, D)$ in $\mathcal{S}_{\omega}$ are nested if and only if they are either comparable with respect to $\leq$, or $A \subseteq D$.

Proof. First assume that the two separations are nested. It is impossible that $B \subseteq C$ and $D \subseteq A$ since the end $\omega$ lies in $B$ and $D$, but not in $C$ and $A$. Hence, if the two separations are not comparable, then we know that $A \subseteq D$ and $C \subseteq B$.

For the converse implication first consider the case that $A \subseteq D$. We want to show that $C \subseteq B$. Assume for a contradiction that there is a vertex $x$ in $C \backslash B$. This vertex must be contained in $A \subseteq D$ and hence in the separator $C \cap D$. By the definition of $\mathcal{S}_{\omega}$ the vertex $x$ must have a neighbour $y$ in $C \backslash D$. Then $y \notin A$ and $x \notin B$, contradicting the fact that the edge $x y$ must lie in either $A$ or $B$, as $(A, B)$ is a separation.

Finally, note that any two separations in $\mathcal{S}_{\omega}$ that are comparable with respect to $\leq$ are obviously nested.

Lemma 3.3. (Analogies with [9, Lemma 4.2]) For each $(A, B) \in \mathcal{S}_{\omega}$ there are only finitely many $(C, D) \in \mathcal{S}_{\omega}$ not nested with $(A, B)$.

Proof. The first step is to show that if $(C, D)$ is not nested with $(A, B)$ then $(C, D)$ separates some vertices $v$ and $w$ in $A \cap B$. Then we show that we may assume that the separation is minimal. Since $A \cap B$ is finite there are only finitely many possibilities for the pair $v, w$ and we can apply Lemma 2.1 to deduce the result. 
First suppose for a contradiction that $(C \backslash D) \cap(A \cap B)$ is empty. Since $C \backslash D$ is connected, it must be a subset of $A \backslash B$ or $B \backslash A$. As every vertex in $C \cap D$ has a neighbour in $C \backslash D$ it follows that $C \subseteq A$ in the first case, whilst $C \subseteq B$ in the second. In both cases $(A, B)$ and $(C, D)$ are nested by Lemma 3.2 . contrary to our assumption. Hence there exists a vertex $v \in(C \backslash D) \cap(A \cap B)$. Note that by letting the separations $(A, B)$ and $(C, D)$ switch roles we see that $(A \backslash B) \cap(C \cap D)$ is also non-empty.

Since the separation $(C, D)$ is in $\mathcal{S}_{\omega}$ there is by Lemma 2.5 a family of $k$ disjoint rays that all have their initial vertices in $C \cap D$. Because $\omega$ lies in $D$, all vertices in these rays, except their initial vertices, are contained in the component of $D \backslash C$ that contains $\omega$. Pick a vertex $v^{\prime}$ from $(A \backslash B) \cap(C \cap D)$. This vertex $v^{\prime}$ is the initial vertex of one of the rays mentioned above. Since $\omega$ lies in $B$ these rays must contain a vertex $w$ from $A \cap B$ and as mentioned above $w$ is contained in the component of $D \backslash C$ that contains $\omega$. Now we have shown that $(C, D)$ separates the two vertices $v$ and $w$. This separation is minimal because $v$ is in $C \backslash D$ and $C \backslash D$ is connected and has $C \cap D$ as it neighbourhood, and $w$ is contained in the component of $G-(C \cap D)$ that contains $\omega$ and that component has the whole of $C \cap D$ as its neighbourhood.

Let $G$ be a one-ended graph whose end $\omega$ is undominated and has finite vertex degree $k$. Recall that by Lemma 3.1 there are no infinite decreasing chains in $\mathcal{S}_{\omega}$ - such a chain would define an end $\mu \neq \omega$, contradicting the assumption that $G$ has only one end. In particular, $\mathcal{S}_{\omega}$ has minimal elements. Assign recursively an ordinal $\alpha(A, B)$ to each $(A, B) \in \mathcal{S}_{\omega}$ by the following method: if $(A, B)$ is minimal (with respect to $\leq$ in $\mathcal{S}_{\omega}$ ) then set $\alpha(A, B)=0$; otherwise define $\alpha(A, B)$ as the smallest ordinal $\beta$ such that $\alpha(C, D)<\beta$ for all separations $(C, D) \in \mathcal{S}_{\omega}$ such that $(C, D)<(A, B)$. For $v \in V(G)$, let $\mathcal{S}_{\omega}(v)$ be the set of those separations $(A, B)$ in $\mathcal{S}_{\omega}$ with $v \in A \cap B$. Now set

$$
\alpha(v)=\sup \left\{\alpha(A, B) \mid(A, B) \in \mathcal{S}_{\omega}(v)\right\} .
$$

If it so happens that $\mathcal{S}_{\omega}(v)$ is empty then $\alpha(v)=0$. For a vertex set $S$, we let $\alpha(S)$ be the supremum over all $\alpha(v)$ with $v \in S$. Note that the functions $\alpha(A, B)$ and $\alpha(v)$ are both invariant under the action of the automorphism group of $G$.

Example 3.4. Below is a construction of a graph where $\alpha$ takes ordinal values that are not natural numbers. However, it is not difficult to show that for a locally finite connected graph the $\alpha$-values are always natural numbers.

We construct a graph $G$ at which $\alpha$ takes values that are not natural numbers. Let $P_{n}=v_{0}^{n}, \ldots, v_{n}^{n}$ be a path of length $n$. We obtain $G$ by taking a ray and identifying its starting vertex $r$ with the vertices $v_{n}^{n}$ for each $n \geq 0$. This graph has only one end $\mu$ and its vertex degree is 1 . For $0 \leq k \leq n-1$ the separation $\left(\left\{v_{0}^{n}, \ldots, v_{k}^{n}\right\}, V(G) \backslash\left\{v_{0}^{n}, \ldots, v_{k-1}^{n}\right\}\right)$ is $\mu$-relevant and its $\alpha$-value is $k$. Hence any separation $(A, B)$ with $r$ (and all the attached paths) in $A$ has $\alpha$-value at least the ordinal $\omega$.

Lemma 3.5. Let $G$ be a graph with only one end $\omega$. Assume that $\omega$ is undominated and has vertex degree $k$. Let $(C, D)$ be in $\mathcal{S}_{\omega}$. Then for all but finitely many vertices $v$ in $C$, we have $\alpha(v) \leq \alpha(C, D)$.

Proof. By Lemma 3.3, there are only finitely many separations in $\mathcal{S}_{\omega}$ that are not nested with $(C, D)$. Let $C^{\prime}$ the set of those vertices in $C \backslash D$ that are not 
in any separator of these finitely many separations. It suffices to show that if $v \in C^{\prime}$ and $(A, B)$ in $\mathcal{S}_{\omega}(v)$ then $\alpha(A, B)<\alpha(C, D)$. Note that the result is trivially true if $\mathcal{S}_{\omega}(v)$ is empty. By the choice of $v$, the separations $(A, B)$ and $(C, D)$ are nested. Since $v$ is in $(C \backslash D) \cap(A \cap B)$, it is not true that $A \subseteq D$ or $B \subseteq D$. Since the end $\omega$ does not lie in the sides $A$ and $C$, it does not lie in the side $A \cup C$ of the separation $(A \cup C, B \cap D)$. Hence it lies in the side $B \cap D$. In particular $B \cap(D \backslash C)$ is nonempty. Thus it is not true that $B \subseteq C$. Looking at the definition of nestedness we see that $A \subseteq C$. Hence $(A, B)<(C, D)$ and thus $\alpha(A, B)<\alpha(C, D)$ and the result follows.

Lemma 3.6. Let $G$ be a graph with only one end $\omega$. Assume that $\omega$ is undominated and has vertex degree $k$. For every separation $(C, D)$ in $\mathcal{S}_{\omega}$, there is a separation $(A, B) \in \mathcal{S}_{\omega}$ such that $C \subseteq A$ and $\alpha(C, D)<\alpha(A, B)$.

Proof. Let $\left\{\left(A_{n}, B_{n}\right)\right\}_{n \geq 0}$ be a sequence of $\omega$-relevant separations as described in Theorem 2.6. Find a separation $(A, B)$ in this sequence such that $C \cap D \subseteq$ $A \backslash B$. Suppose for a contradiction that $C \backslash D$ contains a vertex $x$ from $A \cap B$. There is a ray $R$ that has $x$ as a starting vertex and every other vertex is contained in $B \backslash A$. Because $C \cap D$ contains no vertex from $B$ we see that this ray would be contained in $C \backslash D$, contradicting the assumption that the end $\omega$ lies in $D$. Hence, $C \backslash D$ does not intersect $A \cap B$ and then, since $C \backslash D$ is connected, we conclude that $C \subseteq A$. Thus $\alpha(C, D) \leq \alpha(A, B)$.

By the previous Lemma there are at most finitely many vertices $v$ in $C$ such that $\alpha(v)>\alpha(C, D)$. Suppose for a contradiction that $v$ is such a vertex and there is no value of $n$ such that $\alpha(v)<\alpha\left(A_{n}, B_{n}\right)$. Then we can find a sequence $\left\{\left(C_{n}, D_{n}\right)\right\}_{n \geq 0}$ of separations in $\mathcal{S}_{\omega}(v)$ such that $\alpha\left(C_{1}, D_{1}\right)<$ $\alpha\left(C_{2}, D_{2}\right)<\cdots$ and for every $n$ there is a number $r_{n}$ such $\alpha\left(A_{n}, B_{n}\right)<$ $\alpha\left(C_{n_{r}}, B_{n_{r}}\right)$. By Lemma 3.3 we may assume that for all values of $n$ and $m$ the separations $\left(C_{n}, D_{n}\right)$ and $\left(C_{m}, B_{m}\right)$ are nested. Say that a pair of separations $\left\{\left(C_{n}, D_{n}\right),\left(C_{m}, D_{m}\right)\right\}$ is blue if the separations are comparable with respect to $\leq$ and red otherwise. By Ramsey's Theorem, see e.g. [2, (1.9) on p. 16], there is an infinite set of separations such that all pairs from that set have the same colour. If all pairs from that set were blue then we could find an infinite increasing or a decreasing chain. By Lemma 3.1 (2) there cannot be an infinite descending chain of separations and if there was an infinite increasing chain in $\mathcal{S}_{\omega}(v)$ then, by Lemma 3.1 (3) with the roles of the $A_{i}$ 's and the $B_{i}$ 's reversed, $v$ would be a dominating vertex for the end $\omega$, contrary to assumptions. Hence all pairs from that infinite set must be red and we can conclude that there is an infinite set of separations in the family $\left\{\left(C_{n}, D_{n}\right)\right\}_{n \geq 0}$ such that no two of them are comparable with respect to ordering. We may assume that if $n$ and $m$ are distinct then $\left(C_{n}, D_{n}\right)$ and $\left(C_{m}, D_{m}\right)$ are not comparable and then $C_{n} \backslash D_{n}$ and $C_{m} \backslash D_{m}$ are disjoint. Start by choosing $n$ such that $v \in A_{n} \backslash B_{n}$ and then choose $m$ such that none of the vertices in $A_{n} \cap B_{n}$ is in $C_{m} \backslash D_{m}$. There must be some vertex $u$ that belongs both to $B_{n}$ and $C_{m} \backslash D_{m}$. The set $\left(C_{m} \backslash D_{m}\right) \cup\{v\}$ is connected and thus it contains a $v-u$ path $P$. But $v \in A_{n} \backslash B_{n}$ and $u \in B_{n} \backslash A_{n}$ and the path $P$ contains no vertices from $A_{n} \cap B_{n}$. We have reached a contradiction. Hence our original assumption must be wrong.

Let $X$ be a connected set of vertices which cannot be separated from the end $\omega$ by a separation of order less than $k$. A separation $(A, B) \in \mathcal{S}_{\omega}$ is called $X$ - 
nice, if for every $v \in A \cap B$ we have $\alpha(v)>\alpha(X)$ and there is some $\varphi \in \operatorname{Aut}(G)$ such that $\varphi(X) \subseteq A$ (then we must have $\varphi(X) \subseteq A \backslash B)$. Let $\mathcal{N}(X)$ be the set of all $X$-nice separations in $\mathcal{S}_{\omega}$ which are minimal with respect to $\leq$, i.e. $\mathcal{N}(X)$ contains all $X$-nice separations $(A, B) \in \mathcal{S}_{\omega}$ such that $A$ is minimal with respect to inclusion.

Lemma 3.7. Let $G$ be a graph with only one end $\omega$. Assume that $\omega$ is undominated and has vertex degree $k$.

Suppose $(X, Y) \in \mathcal{S}_{\omega}$. Then $\mathcal{N}(X)$ is non-empty. For each automorphism $\varphi$ of $G$ there is a unique element $(A, B)$ in $\mathcal{N}(X)$ such that $\varphi(X) \subseteq A$. If $(A, B)$ and $(C, D)$ are not equal and in $\mathcal{N}(X)$, then $A \subseteq D$ and $C \subseteq B$. Furthermore, any two elements of $\mathcal{N}(X)$ can be mapped onto each other by an automorphism.

Proof. The existence of an $X$-nice separation follows from Lemma 3.6. Minimal such separations exist because by Lemma 3.1 an infinite descending chain would imply that $G$ had another end $\mu \neq \omega$.

Let $(A, B)$ and $(C, D)$ be elements of $\mathcal{N}(X)$. Suppose $\varphi(X) \subseteq A$ and $\psi(X) \subseteq$ $C$, where $\varphi, \psi \in \operatorname{Aut}(G)$. Note that $\varphi(X)$ is disjoint from $C \cap D$ because $\alpha(\varphi(X))=\alpha(X)$, which is strictly less than $\alpha(v)$ for any $v \in C \cap D$. Hence it is a subset of either $C \backslash D$ or $D \backslash C$. We next prove that if $(A, B)$ and $(C, D)$ are not equal, then $A \subseteq D$ and $C \subseteq B$.

First we consider the case that $\varphi(X)$ is a subset of $C \backslash D$. Our aim is to show that $(A, B)$ and $(C, D)$ are equal. This also implies that $(A, B)$ is the unique element in $\mathcal{N}(X)$ such that $\varphi(X) \subseteq A$. Our strategy will be to construct a $X$-nice separation that is $\leq$ to both of them and by minimality of $(A, B)$ and $(C, D)$ we will conclude that it must be equal to both of them. Note that $\varphi(X)$ is included in $(C \backslash D) \cap(A \backslash B)$. Let $A^{\prime}$ be the connected component of $(C \backslash D) \cap(A \backslash B)$ that contains the connected set $\varphi(X)$ together with the separator of $(A \cap C, B \cup D)$. Let $B^{\prime}$ be the union of $B \cup D$ with the other components of $(C \backslash D) \cap(A \backslash B)$.

Next we show that the separation $\left(A^{\prime}, B^{\prime}\right)$ is in $\mathcal{N}(X)$. Since the end $\omega$ lies in $B \cap D$, this vertex set is infinite. Because $(A, B)$ is in $\mathcal{S}_{\omega}$, the separation $(A \cup C, B \cap D)$ has order at least $k$. Hence by Lemma 2.2 the separation $(A \cap C, B \cup D)$ has order at most $k$. The property that $X$ cannot be separated from $\omega$ by fewer than $k$ vertices implies that the separation $\left(A^{\prime}, B^{\prime}\right)$ has order precisely $k$. Also, every vertex of the separator of $\left(A^{\prime}, B^{\prime}\right)$ has a neighbour in $A^{\prime} \backslash B^{\prime}$ and in $B^{\prime} \backslash A^{\prime}$. Clearly $\omega$ lies in $B^{\prime}$ and there is no separation $\left(C^{\prime}, D^{\prime}\right)$ of order less than $k$ such that $A^{\prime} \subseteq C^{\prime}$ and $\omega$ lies in $D^{\prime}$ as $(X, Y) \in \mathcal{S}_{\omega}$. Hence $\left(A^{\prime}, B^{\prime}\right)$ is in $\mathcal{S}_{\omega}$ and thus it is in $\mathcal{N}(X)$ as $A^{\prime} \subseteq A$. Since $A^{\prime} \subseteq A$, it must be that $A^{\prime}=A$ by the minimality of $(A, B)$. Similarly, $A^{\prime}=C$. Thus $A=C$ and so $(A, B)=(C, D)$. This completes the case when $\varphi(X)$ is a subset of $C \backslash D$.

So we may assume that $\varphi(X) \subseteq D \backslash C$, and by symmetry that $\psi(X) \subseteq B \backslash A$. Consider the separations $(A \cap D, B \cup C)$ and $(B \cap C, A \cup D)$. They must have order at least $k$ because $\varphi(X) \subseteq A \cap D, \omega \in B \cup C$ and $\psi(X) \subseteq B \cap C, \omega \in A \cup D$. So they must have order precisely $k$ by Lemma 2.2. Let $A^{\prime}$ be the component of $G-(B \cup C)$ that contains $\varphi(X)$ together with the separator of $(A \cap D, B \cup C)$. Let $B^{\prime}$ be the union of $B \cup C$ with the other components. Similar as in the last case we show that $\left(A^{\prime}, B^{\prime}\right)$ is in $\mathcal{N}(X)$. By the minimality of $(A, B)$ it must be that $A \subseteq D$. The above argument with the separation $(B \cap C, A \cup D)$ in place of $(A \cap D, B \cup C)$ yields that $C \subseteq B$. This completes the proof that if $(A, B)$ and $(C, D)$ are not equal and in $\overline{\mathcal{N}}(X)$, then $(A, B)$ and $(C, D)$ are nested. 
By the above there is for each $\varphi \in \operatorname{Aut}(G)$ a unique separation $\left(A_{\varphi}, B_{\varphi}\right) \in$ $\mathcal{N}(X)$ such that $\varphi(X) \subseteq A_{\varphi}$. If we apply $\varphi^{-1}$ to this separation we must obtain the unique separation $(A, B) \in \mathcal{N}(X)$ such that $X \subseteq A$. Hence any separation of $\mathcal{N}(X)$ can be mapped by an automorphism to every other separation in $\mathcal{N}(X)$.

Theorem 3.8. Let $G$ be a connected graph with only one end $\omega$, which is undominated and has finite vertex degree $k$. Then there is a nested set $\mathcal{S}$ of $\omega$-relevant separations of $G$ that is $\operatorname{Aut}(G)$-invariant. And there is a 1-ended tree $T$ and a bijection between the edge set of $T$ and $\mathcal{S}$ such that the natural action of $\operatorname{Aut}(G)$ on $S$ induces an action on $T$ by automorphisms.

Proof. Pick some $\omega$-relevant separation $\left(A_{0}, B_{0}\right)$. Define a sequence $\left(A_{n}, B_{n}\right)$ of separations as follows. For $n \in \mathbb{N}_{>0}$ pick $\left(A_{n}, B_{n}\right) \in \mathcal{N}\left(A_{n-1}\right)$ such that $A_{n-1} \subsetneq A_{n}$, which is possible by Lemma 3.7. Observe that the sequence of separations $\left(A_{n}, B_{n}\right)$ has the same properties as the sequence in Theorem 2.6.

Now let

$$
\mathcal{S}=\left\{\left(\varphi\left(A_{n}\right), \varphi\left(B_{n}\right)\right) \mid n \in \mathbb{N}_{>0}, \varphi \in \operatorname{Aut}(G)\right\} .
$$

Note that $\left(A_{0}, B_{0}\right)$ is not an element in $\mathcal{S}$.

First we prove that $\mathcal{S}$ is nested. Let $\left(\varphi\left(A_{n}\right), \varphi\left(B_{n}\right)\right)$ and $\left(\psi\left(A_{m}\right), \psi\left(B_{m}\right)\right)$ be two different elements of $\mathcal{S}$ (here $\varphi$ and $\psi$ are automorphisms of $G$ ). If $m=n$ then they are nested by Lemma 3.7, since they both are elements of $\mathcal{N}\left(A_{n-1}\right)$. Hence assume without loss of generality that $n<m$. If $\varphi\left(A_{m}\right)=\psi\left(A_{m}\right)$ then $\varphi\left(A_{n}\right) \subseteq \varphi\left(A_{m}\right)=\psi\left(A_{m}\right)$ which implies that the two separations are nested. Otherwise by Lemma 3.7 we have $\varphi\left(A_{n}\right) \subseteq \varphi\left(A_{m}\right) \subseteq \psi\left(B_{m}\right)$, also showing nestedness, by Lemma 3.2 .

Next we construct a directed graph $T_{+}$. We define $T_{+}$as follows. Its vertex set is $\mathcal{S}$. We add a directed edge from $\left(\varphi\left(A_{n}\right), \varphi\left(B_{n}\right)\right)$ to $\left(\psi\left(A_{n+1}\right), \psi\left(B_{n+1}\right)\right)$ if $\varphi\left(A_{n}\right)$ is a subset of $\psi\left(A_{n+1}\right)$. By Lemma 3.7. each vertex has outdegree at most one. And by the construction of $\mathcal{S}$ it has outdegree at least one.

The next step is to show that the graph is connected. Let $(C, D)=\varphi\left(A_{n}, B_{n}\right)$ be a vertex in $T_{+}$. Find an $m$ such that $C \subseteq A_{m} \backslash B_{m}$. Suppose for a contradiction that $\left(\varphi\left(A_{m}\right), \varphi\left(B_{m}\right)\right) \neq\left(A_{m}, B_{m}\right)$. Both $\left(\varphi\left(A_{m}\right), \varphi\left(B_{m}\right)\right)$ and $\left(A_{m}, B_{m}\right)$ are in $\mathcal{N}(X)$. By Lemma $3.7 \varphi\left(A_{m}\right) \subseteq B_{m}$. Thus $\varphi\left(A_{m}\right)$ is empty. This is a contradiction to the assumption that $\left(A_{m}, B_{m}\right)$ is a proper separation. Now we see that

$$
\left(A_{m}, B_{m}\right)=\left(\varphi\left(A_{m}\right), \varphi\left(B_{m}\right)\right),\left(\varphi\left(A_{m-1}\right), \varphi\left(B_{m-1}\right)\right), \ldots,\left(\varphi\left(A_{n}\right), \varphi\left(B_{n}\right)\right)=(C, D)
$$

is a path in $T_{+}$from $\left(A_{m}, B_{m}\right)$ to $(C, D)$. Thus every vertex in $T_{+}$is in the same connected component as some vertex $\left(A_{m}, B_{m}\right)$ and since they all belong to the same component we deduce that $T_{+}$is connected. Hence the corresponding undirected graph $T$ is a tree.

The map that sends $\left(\varphi\left(A_{n}\right), \varphi\left(B_{n}\right)\right)$ to the edge with endvertices $\left(\varphi\left(A_{n}\right), \varphi\left(B_{n}\right)\right)$ and $\left(\psi\left(A_{n+1}\right), \psi\left(B_{n+1}\right)\right)$ is clearly a bijection. If the ray $\left(A_{1}, B_{1}\right),\left(A_{2}, B_{2}\right), \ldots$ is removed from $T$ then what remains of $T$ is clearly rayless and thus the tree $T$ is one-ended.

The statement about the action of $\operatorname{Aut}(G)$ on $T$ follows easily since the properties used to define $T$ are invariant under $\operatorname{Aut}(G)$. 
A tree-decomposition of a graph $G$ consists of a tree $T$ and a family $\left(P_{t}\right)_{t \in V(T)}$ of subsets of $V(G)$, one for each vertex of $T$ such that

(T1) $V(G)=\bigcup_{t \in V(T)} P_{t}$,

(T2) for every edge $e \in E(G)$ there is $t \in V(T)$ such that both endpoints of $e$ lie in $P_{t}$, and

(T3) $P_{t_{1}} \cap P_{t_{3}} \subseteq P_{t_{2}}$ whenever $t_{2}$ lies on the unique path connecting $t_{1}$ and $t_{3}$ in $T$.

The tree $T$ is called decomposition tree, the sets $P_{t}$ are called the parts of the tree-decomposition.

We associate to an edge $e=s t$ of the decomposition tree a separation of $G$ as follows. Removing $e$ from $T$ yields two components $T_{s}$ and $T_{t}$. Let $X_{s}=$ $\bigcup_{u \in T_{s}} P_{u}$ and $X_{t}=\bigcup_{u \in T_{t}} P_{u}$. If $X_{s} \backslash X_{t}$ and $X_{t} \backslash X_{s}$ are non-empty (this will be the case for all tree-decompositions considered in this paper), then $\left(X_{s}, X_{t}\right)$ is a proper separation of $G$. Clearly, the set of all separations associated to edges of a decomposition tree is nested.

The separators $A \cap B$ of the separations associated to edges of a decomposition tree are called adhesion sets. The supremum of the sizes of adhesion sets is called the adhesion of the tree-decomposition. The tree-decompositions constructed in this paper all have finite adhesion.

Given a graph $G$ with only one end $\omega$ and a tree-decomposition $\left(T, P_{t} \mid t \in\right.$ $V(T))$ of $G$ of finite adhesion, then $\left(T, P_{t} \mid t \in V(T)\right)$ displays $\omega$ if firstly the decomposition tree $T$ has only one end; call it $\mu$. And secondly for any edge $s t$ of $T$ with $\mu$ in $T_{t}$, the associated separation $\left(X_{s}, X_{t}\right)$ has the property that $\omega$ lies in $X_{t}$.

A tree-decomposition is $\operatorname{Aut}(G)$-invariant if the set $S$ of separations associated to it is closed by the natural action of $\operatorname{Aut}(G)$ on $S$. The following implies Theorem 1.1

Theorem 3.9. Let $G$ be a connected graph with only one end $\omega$, which is undominated and has finite vertex degree $k$. Then $G$ has a tree-decomposition $\left(T, P_{t} \mid t \in V(T)\right)$ of adhesion $k$ that displays $\omega$ and is $\operatorname{Aut}(G)$-invariant.

Proof. We follow the notation of the proof of Theorem 3.8

Given a vertex $t$ of $T_{+}$, the inward neighbourhood of $t$, denoted by $N_{+}(t)$, is the set of vertices $u$ of $T_{+}$such that there is a directed edge from $u$ to $t$ in $T_{+}$. Recall that the vertices of $T_{+}$are (in bijection with) separations; we refer to the separation associated to the vertex $t$ by $\left(A_{t}, B_{t}\right)$. Given a vertex $t$, we let $P_{t}=A_{t} \backslash \bigcup_{u \in N_{+}(t)}\left(A_{u} \backslash B_{u}\right)$.

It is straightforward that $\left(T, P_{t} \mid t \in V(T)\right)$ is a tree-decomposition of adhesion $k$ (whose set of associated separations is $\mathcal{S} \cup\{(B, A) \mid(A, B) \in \mathcal{S}\}$ ). It is not hard to see that $\left(T, P_{t} \mid t \in V(T)\right)$ displays $\omega$ and is $\operatorname{Aut}(G)$-invariant.

Example 3.10. In this example we construct a one-ended graph $G$ whose end is dominated and has vertex degree 1 , but the graph $G$ has no tree-decomposition of finite adhesion that is invariant under the group of automorphisms and whose decomposition tree is one-ended. We obtain $G$ from the canopy tree by adding a new vertex adjacent to all the leaves of the canopy tree. Then we add infinitely many vertices of degree one only incident to that new vertex, see Figure 2 . 


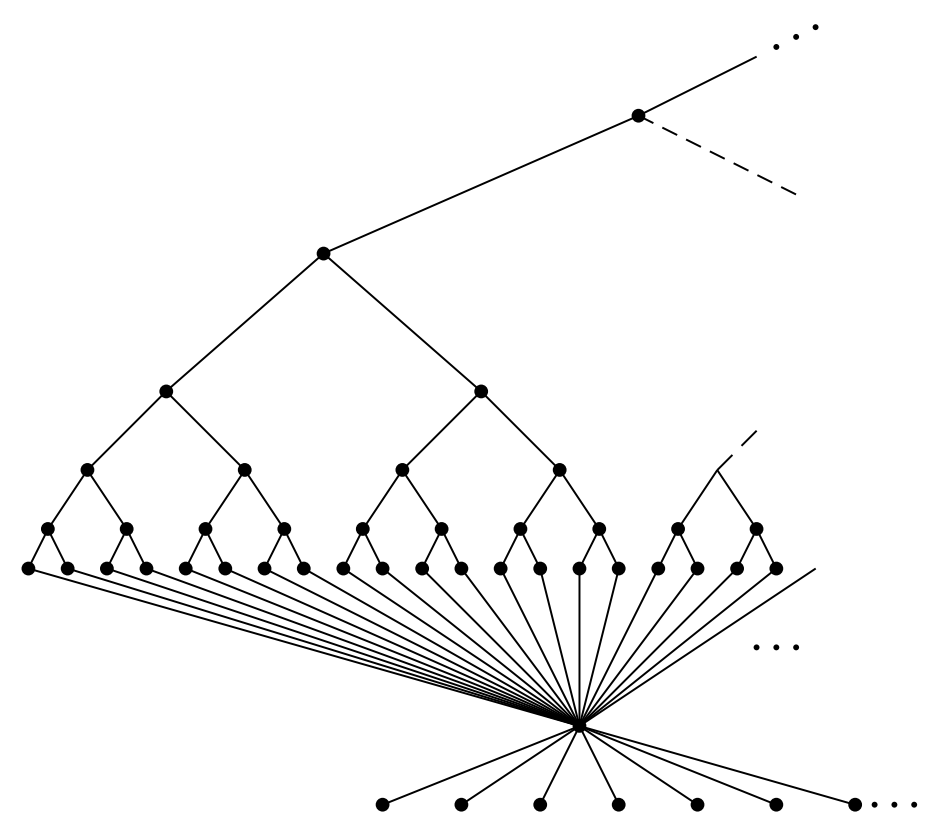

Figure 2: A graph with no $\operatorname{Aut}(G)$-invariant tree-decomposition of finite adhesion.

Suppose for a contradiction that $G$ has a tree-decomposition $\left(T, P_{t} \mid t \in\right.$ $V(T)$ ) of finite adhesion that is invariant under the group of automorphisms and such that $T$ is one-ended.

There cannot be a single part $P_{t}$ that contains a ray of the canopy tree. To see that first note that there cannot be two such parts by the assumption of finite adhesion. Hence any such part would contain all vertices of the canopy tree from a certain level onwards. This is not possible by finite adhesion.

Having shown that there cannot be a single part $P_{t}$ that contains a ray of the canopy tree, it must be that every part $P_{t}$ with $t$ near enough to the end of $T$ contains a vertex of the canopy tree.

Our aim is to show that any vertex $u$ of degree 1 is in all parts. Suppose not for a contradiction. Then since $T$ is one-ended, there is a vertex $t$ of $T$ such that $t$ separates in $T$ all vertices $s$ with $u \in P_{s}$ from the end of $T$. We pick $t$ high enough in $T$ such that there is a vertex $v$ of the canopy tree in $P_{t}$. If $P_{t}$ contained all vertices of the orbit of $v$, then $P_{t}$ together with all parts $P_{s}$, where $s$ has some fixed bounded distance from $t$ in $T$, would contain a ray. This is impossible; the proof is similar as that that $P_{t}$ cannot contain a ray. Hence there is a vertex $v^{\prime}$ in the orbit of $v$ that is not in $P_{t}$. Take an automorphism of $G$ that fixes $u$ and moves $v$ to $v^{\prime}$. As the tree-decomposition is $\operatorname{Aut}(G)$-invariant, $T$ has a vertex $s$ such that $u, v^{\prime} \in P_{s}$ but $v \notin P_{s}$. Since $T$ is $\operatorname{Aut}(G)$-invariant and one-ended, $t$ does not separate $s$ from the end of $T$. This is a contradiction as $u \in P_{s}$.

Hence $u$ must be in all parts. As $u$ was arbitrary, every vertex of degree one must be in every part. So the tree-decomposition does not have finite adhesion. This is the desired contradiction. Hence such a tree-decomposition does not exist. 


\section{A dichotomy result for automorphism groups}

Before we turn to a proof of Theorem 1.2, we state a few helpful auxiliary results. The following lemma can be seen as a consequence of [17, Lemma 7], but for completeness a direct proof is provided in Appendix B.

Lemma 4.1. If $T$ is a one-ended tree and $R$ is a ray in $T$, then every automorphism of $T$ fixes some tail of $R$ pointwise.

The next result is Lemma 3 in [17. For completeness a proof is included in Appendix C.

Lemma 4.2. The pointwise (and hence also the setwise) stabiliser of a finite set of vertices in the automorphism group of a rayless graph is either finite or contains at least $2^{\aleph_{0}}$ many elements.

The next result is an extension of Lemma 4.2 to one-ended graphs where the end has finite vertex degree.

Lemma 4.3. Let $G$ be a graph with only one end $\omega$. Assume that $\omega$ has finite vertex degree $k$. Let $X$ be a finite set of vertices in $G$ that contains all the vertices that dominate the end. If the graph $G-X$ is connected then the pointwise stabiliser of $X$ in $\operatorname{Aut}(G)$ is either finite or contains at least $2^{\aleph_{0}}$ many elements.

Proof. Denote by $\Gamma$ the pointwise stabiliser of $X$ in $\operatorname{Aut}(G)$. If $\Gamma$ is finite, then there is nothing to show, hence assume that $\Gamma$ is infinite.

Consider a nested $\operatorname{Aut}(G-X)$-invariant set of $\omega$-relevant separations of $G-X$ as in Theorem 3.8 and a tree $T$ built from this set in the way described. Clearly $\Gamma$ gives rise to a subgroup of $\operatorname{Aut}(G-X)$ whence this nested set is $\Gamma$-invariant. Adding $X$ to both sides of every separation in $\mathcal{S}$ gives rise to a new $\Gamma$ invariant set $\mathcal{S}$ of nested separations such that each separation has order $k+|X|$. The tree we get from $\mathcal{S}$ is the same as $T$. From now on we will work with $\mathcal{S}$.

Every element $\gamma \in \Gamma$ induces an automorphism of $T$. Note that this canonical action of $\Gamma$ on $T$ is in general not faithful, i.e. it is possible that different elements of $\Gamma$ induce the same automorphism of $T$.

Let $R$ be a ray in $T$ and let $\left(e_{n}\right)_{n \in \mathbb{N}}$ be the family of edges of $R$ (in the order in which they appear on $R$ ). Let $\left(A_{n}, B_{n}\right)$ be the separation of $G$ corresponding to $e_{n}$. Denote by $\Gamma_{n}$ the stabiliser of $e_{n}$ in $\Gamma$. By Lemma 4.1 every automorphism of $T$ (and hence also every $\gamma \in \Gamma$ ) fixes some tail of $R$, so $\Gamma_{n}$ is non-trivial for large enough $n$. Furthermore, $\Gamma_{n}$ is a subgroup of $\Gamma_{m}$ whenever $n \leq m$.

We claim that for all but finitely many $n$, we have at least one non-trivial $\gamma$ in the pointwise stabiliser of $B_{n}$. To see this, let $\gamma_{1}, \ldots, \gamma_{(k+|X|) !+1}$ be a set of $(k+|X|) !+1$ different non-trivial automorphisms in $\Gamma$. Choose $n$ large enough such that they all are contained in $\Gamma_{n}$ and act differently on $A_{n}$. By a simple pigeon hole argument, at least two of them, $\gamma_{1}$ and $\gamma_{2}$ say, have the same action on $A_{n} \cap B_{n}$. Then $\gamma_{1} \circ \gamma_{2}^{-1}$ is an automorphism which fixes $A_{n} \cap B_{n}$ pointwise, and fixes $A_{n}$ setwise but not pointwise. Now, using the independence property from Section 2.2 we can define an automorphism

$$
\gamma(x)= \begin{cases}\gamma_{1} \circ \gamma_{2}^{-1}(x) & \text { if } x \in A_{n} \backslash B_{n} \\ x & \text { if } x \in B_{n}\end{cases}
$$

with the desired properties. 
Note that the subgroup leaving $A_{n}$ invariant in the pointwise stabiliser of $B_{n}$ in $\Gamma$ induces the same permutation group on the rayless graph induced by $A_{n}$ in $G$ as does the subgroup leaving $A_{n}$ invariant in the pointwise stabiliser of $A_{n} \cap B_{n}$. Hence, if there is $n \in \mathbb{N}$ such that the pointwise stabiliser of $B_{n}$ in $\Gamma$ is infinite, then this stabiliser contains at least $2^{\aleph_{0}}$ many elements by Lemma 4.2 .

So (by passing to a tail of $R$ ) we may assume that the pointwise stabiliser of $B_{n}$ is a finite but non-trivial subgroup of $\Gamma$ for every $n \in N$.

Next we claim that for every $n$ there is a non-trivial automorphism in the pointwise stabiliser of $A_{n}$. If not, then $\Gamma_{n}$ is finite and we choose $\sigma \in \Gamma \backslash \Gamma_{n}$. For an edge $e$ of $T$, denote by $T_{e}$ the component of $T-e$ which does not contain the end of $T$. Clearly $\sigma\left(T_{e}\right)=T_{\sigma(e)}$ for every edge $e$. In particular, if $e=e_{m}$ is the last edge of $R$ which is not fixed by $\sigma$, then clearly $\sigma\left(T_{e}\right) \subseteq T-T_{e}$. Furthermore $n<m$, so $A_{n} \subseteq A_{m}$, and $B_{m} \subseteq B_{n}$. Hence $\sigma\left(A_{n}\right) \subseteq \sigma\left(A_{m}\right) \subseteq B_{m} \subseteq B_{n}$. Now let $\gamma$ be a nontrivial automorphism in the pointwise stabiliser of $B_{n}$. Then $\sigma^{-1} \circ \gamma \circ \sigma$ is easily seen to be a nontrivial element of the pointwise stabiliser of $A_{n}$ : for $a \in A_{n}$ we have

$$
\sigma^{-1} \circ \gamma \circ \sigma(a)=\sigma^{-1} \circ \sigma(a)=a
$$

since $\sigma(a) \in B_{n}$ is fixed by $\gamma$.

Now define an infinite sequence $\left(\gamma_{k}\right)_{k \in \mathbb{N}}$ of elements of $\Gamma$ as follows. Pick a nontrivial $\gamma_{1}$ in the pointwise stabiliser of $A_{1}$. Assume that $\gamma_{i}$ has been defined for $i<k$, then let $n_{k}$ be such that $\gamma_{i}$ acts non-trivially on $A_{n_{k}}$ for all $i<k$ and pick a nontrivial element $\gamma_{k}$ in the pointwise stabiliser of $A_{n_{k}}$. For an infinite 0-1-sequence $\left(r_{j}\right)_{j \geq 1}$, define

$$
\psi_{i}=\gamma_{i}^{r_{i}} \circ \gamma_{i-1}^{r_{i-1}} \circ \cdots \circ \gamma_{1}^{r_{1}}
$$

in other words, $\psi_{n}$ is the composition of all $\gamma_{j}$ with $j \leq n$ and $r_{j}=1$. Finally define $\psi$ to be the limit of the $\psi_{n}$ in the topology of pointwise convergence. This limit exists, because for $j>i$ the restriction $\psi_{i}$ and $\psi_{j}$ to $A_{n_{i}}$ coincide, and the $A_{n_{i}}$ exhaust $V(G)$. By Lemma 2.8, $\psi$ is contained in $\operatorname{Aut}(G)$ and is also in $\Gamma \subseteq \operatorname{Aut}(G)$ because every $\psi_{i}$ stabilises $X$ pointwise.

Finally assume that we have two different 0 -1-sequences $\left(r_{j}\right)_{j \geq 1}$ and $\left(r_{j}^{\prime}\right)_{j \geq 1}$ and let $\left(\psi_{j}\right)_{j \geq 1}$ and $\left(\psi_{j}^{\prime}\right)_{j \geq 1}$ be the corresponding sequences of automorphisms. If $l$ is the first index such that $r_{l} \neq r_{l}^{\prime}$ then the restrictions of $\psi_{l}$ and $\psi_{l}^{\prime}$ (and hence also of $\psi_{i}$ and $\psi_{i}^{\prime}$ for $i>l$ ) to $A_{n_{l}}$ differ. Hence different 0-1-sequences give different elements of $\Gamma$ and $\Gamma$ contains at least $2^{\aleph_{0}}$ many elements.

Theorem 1.2. Let $G$ be a graph with one end which has finite vertex degree. Then $\operatorname{Aut}(G)$ is either finite or has at least $2^{\aleph_{0}}$ many elements.

Proof. Let $X$ be the set of vertices which dominate $\omega$. This set is possibly empty and by Lemma 2.3 it is finite. Every automorphism stabilises $X$ setwise. Therefore the pointwise stabiliser of $X$ is a normal subgroup of $\operatorname{Aut}(G)$ with finite index. So it suffices to show that the conclusion of Theorem 1.2 holds for the stabiliser $\Gamma$ of $X$.

For every component $C$ of $G-X$ let $\Gamma_{C}$ be the pointwise stabiliser of $X$ in $\operatorname{Aut}(C \cup X)$. Then $\Gamma_{C}$ is either finite or contains at least $2^{\aleph_{0}}$ many elements by Lemma 4.2 and Lemma 4.3. If $\left|\Gamma_{C}\right|=2^{\aleph_{0}}$ for some component $C$ then we need do no more. So assume that all the groups $\Gamma_{C}$ are finite. The same argument as used towards the end of the proof of Lemma 4.2 (see Appendix C) now shows that either $\Gamma$ is finite or has at least cardinality $2^{\aleph_{0}}$. 
As a corollary we can answer a question posed by Boutin and Imrich in 1 . In order to state this question, we first need some notation. For a vertex $v$ in a graph $G$ we define $B_{v}(n)$, the ball of radius $n$ centered at $v$, as the set of all vertices in $G$ in distance at most $n$ from $v$. We also define $S_{v}(n)$, the sphere of radius $n$ centered at $v$, as the set of all vertices in $G$ in distance exactly $n$ from $v$. A connected locally finite graph is said to have linear growth if there is a constant $c$ such that $\left|B_{v}(n)\right| \leq c n$ for all $n=1,2, \ldots$. It is an easy exercise to show that the property of having linear growth does not depend on the choice of the vertex $v$.

In relation to their work on the distinguishing cost of graphs Boutin and Imrich [1] ask whether there exist one-ended locally finite graphs that has linear growth and countably infinite automorphism group.

If $G$ is a locally finite graph with linear growth and $v$ is a vertex in $G$ then there is a constant $k$ such that $\left|S_{v}(n)\right|=k$ for infinitely many values of $n$. (This is observed by Boutin and Imrich in their paper [1, Fact 2 in the proof of Proposition 13].) From this we deduce that the vertex-degree of an end of $G$ is at most equal to $k$, since each ray in $G$ must pass through all but finitely many of the spheres $S_{v}(n)$. Using Theorem 1.2 one can now give a negative answer to the above question.

Theorem 4.4. If $G$ is a connected locally finite graph with one end and linear growth, then the automorphism group of $G$ is either finite or contains exactly $2^{\aleph_{0}}$ many elements.

Proof. Since $G$ is locally finite and connected, the graph $G$ is countable. Hence the automorphism group cannot contain more than $2^{\aleph_{0}}$ many elements. Furthermore linear growth implies that all ends must have finite vertex degree, hence we can apply Theorem 1.2 .

In particular a connected graph with linear growth and a countably infinite autormorphism group cannot have one end. Thus one can strengthen [1, Theorem 22] and get:

Theorem 4.5. (Cf. [1, Theorem 22]) Every locally finite connected graph with linear growth and countably infinite automorphism group has 2 ends.

Furthermore one can in [1, Theorem 18] remove the assumption that the graph is 2-ended, since it is implied by the other assumptions.

\section{Ends of quasi-transitive graphs}

Finally, another application was pointed out to the authors by Matthias Hamann. Recall that a graph is called transitive, if all vertices lie in the same orbit under the automorphism group, and quasi-transitive (or almost-transitive), if there are only finitely many orbits on the vertices.

The groundwork for the study of automorphisms of infinite graphs was laid in the 1973 paper of Halin [13. Among the results there is a classification of automorphisms of a connected infinite graph, see [13, Sections 5, 6 and 7]. Type 1 automorphisms, to use Halin's terminology, leave a finite set of vertices invariant. An automorphism is said to be of type 2 if it is not of type 1 . Type 2 automorphism are of two kinds, the first kind fixes precisely one end which 
is then thick (i.e. has infinite vertex degree) and the second kind fixes precisely two ends which are then both thin (i.e. have finite vertex degrees). In Halin's paper these results are stated with the additional assumption that the graph is locally finite but the classification remains true without this assumption.

It is a well known fact that a connected, transitive graph has either 1, 2, or infinitely many ends (follows for locally finite graphs from Halin's paper [11, Satz 2] and for the general case see [7, Corollary 4]). It is a consequence of a result of Jung [20 that if such a graph has more than one end then there is a type 2 automorphism that fixes precisely two ends and thus the graph has at least two thin ends. In particular, in the two-ended case both of the ends must be thin. Contrary to this, we deduce from Theorem 3.8 that the end of a one-ended transitive graph is always thick. This even holds in the more general case of quasi-transitive graphs. This was proved for locally finite graphs by Thomassen [23, Proposition 5.6]. A variant of this result for metric ends was proved by Krön and Möller in [21, Theorem 4.6].

Theorem 5.1. If $G$ is a one-ended, quasi-transitive graph, then the unique end is thick.

For the proof we need the following auxiliary result.

Proposition 5.2. There is no one-ended quasi-transitive tree.

Proof. Assume that $T$ is a quasi-transitive tree and that $R$ is a ray in $T$. Then there is an edge-orbit under $\operatorname{Aut}(T)$ containing infinitely many edges of $R$. Contract all edges not in this orbit to obtain a tree $T^{\prime}$ whose automorphism group acts transitively on edges. Clearly, every end of $T^{\prime}$ corresponds to an end of $T$ (there may be more ends of $T$ which we contracted). But edge transitive trees must be either regular, or bi-regular. Hence $T^{\prime}$, and thus also $T$, has at least 2 ends.

Proof of Theorem 5.1. Assume for a contradiction that $G$ is a quasi-transitive, one-ended graph whose end is thin.

If the end $\omega$ is dominated, then remove all vertices which dominate it and only keep the component $C$ in which $\omega$ lies. The resulting graph is still quasitransitive since $C$ must be stabilised setwise by every automorphism. Furthermore, the degree of $\omega$ does not increase by deleting parts of the graph. Hence we can without loss of generality assume that the end of the counterexample $G$ is undominated.

Now apply Theorem 3.8 to $G$. This gives a nested set $\mathcal{S}$ of separations which is invariant under automorphisms - in particular, there are only finitely many orbits of $\mathcal{S}$ under the action of $\operatorname{Aut}(G)$. Theorem 3.8 further tells us that there is a bijection between $\mathcal{S}$ and the edges of a one-ended tree $T$ such that the action of $\operatorname{Aut}(G)$ on $\mathcal{S}$ induces an action on $T$ by automorphisms. Hence $T$ is a quasi-transitive one-ended tree, which contradicts Proposition 5.2

\section{A Appendix}

We say that a vertex $v$ dominates a ray $L$ if there are infinitely many $v-L$ paths, any two only having $v$ as a common vertex. It follows from the definition of an end that if a vertex domintes one ray belonging to an end then it dominates every ray belonging to that end and dominates the end. 
Proof of Lemma 2.3. Assume that the set $X$ of dominating vertices is infinite. By the above we can assume that there is a ray $R$ and infinitely many vertices $x_{1}, x_{2}, \ldots$ that dominate $R$ in $G$. We show that $G$ must then contain a subdivision of the complete graph on $x_{1}, x_{2}, \ldots$. Start by taking vertices $v_{1}$ and $v_{2}$ on $R_{1}$ such that there are disjoint $x_{1}-v_{1}$ and $x_{2}-v_{2}$ paths. Then we find vertices $w_{1}$ and $w_{2}$ furher along the ray $R_{1}$ such that there are disjoint $x_{1}-w_{1}$ and $x_{3}-w_{3}$ paths and still further along we find vertices $u_{2}$ and $u_{3}$ such that there are disjoint $x_{2}-u_{2}$ and $x_{3}-u_{3}$ paths. Adding the relevant segments of $R$ we find $x_{1}-x_{2}, x_{1}-x_{3}$ and $x_{2}-x_{3}$ paths having at most their endvertices in common. The subgraph of $G$ consisting of these three paths is thus a subdivision of the complete graph on three vertices. Using induction we can find an increasing sequence of subgraphs $H_{n}$ of $G$ that contains the vertices $x_{1}, x_{2}, \ldots, x_{n}$ and also paths $P_{i j}$ linking $x_{i}$ and $x_{j}$ such that any two such paths have at most their end vertices in common. The subgraph $H_{n}$ is a subdivision of the complete graph on $n$-vertices. The subgraph $H=\bigcup_{i=1}^{\infty} H_{i}$ is a subdivision of the complete graph on (countably) infinite set of vertices and contains an infinite family of pairwise disjoint rays that all belong to the end $\omega$. This contradicts our assumptions and we conclude that $T$ must be finite.

A ray decomposition 4 of adhesion $m$ of a graph $G$ consists of subgraphs $G_{1}, G_{2}, \ldots$ such that:

1. $G=\bigcup_{i=1}^{\infty} G_{i}$;

2. if $T_{n+1}=\left(\bigcup_{i=1}^{n} G_{i}\right) \cap G_{n+1}$ then $\left|T_{n+1}\right|=m$ and $T_{n+1} \subseteq G_{n} \backslash\left(\bigcup_{i=1}^{n-1} G_{i}\right)$ for $n=1,2, \ldots$;

3. for each value of $n=1,2, \ldots$ there are $m$ pairwise disjoint paths in $G_{n+1}$ that have their initial vertices in $T_{n+1}$ and teminal vertices in $T_{n+2}$;

4. none of the subgraphs $G_{i}$ contains a ray.

The following Menger-type result is used by Halin in his proof of 12, Satz 2]. In the proof we also use ideas from another one of Halin's papers [10, Proof of Satz 3].

Theorem A.1. Let $G$ be a locally finite connected graph with the property that $G$ contains a family of $m$ pairwise disjoint rays but there is no such family of $m+1$ pairwise disjoint rays. Then there is in $G$ a family of pairwise disjoint separators $T_{1}, T_{2}, \ldots$ such that each contains precisely $m$ vertices and a ray in $G$ must for some $n_{0}$ intersects all the sets $T_{n}$ for $n \geq n_{0}$.

Proof. Fix a reference vertex $v_{0}$ in $G$. Let $E_{j}$ denote the set of vertices in distance precisely $j$ from $v_{0}$. Define also $B_{i}$ as the set of vertices in distance at most $i$ from $v_{0}$. For numbers $i$ and $j$ such that $i+1<j$ we construct a new graph $H_{i j}$ such that we start with the subgraph of $G$ induces by $B_{j}$, then we remove $B_{i}$ but add a new vertex $a$ that has as its neighbourhood the set $\partial B_{i}$ (for a set $C$ of vertices $\partial C$ denotes the set of vertices that are not in $C$ but are adjacent to some vertex in $C$ ) and we also add a new vertex $b$ that has every vertex in $\partial\left(G \backslash B_{j}\right)$ as its neighbour. Since $G$ is assumed to be locally finite the

\footnotetext{
${ }^{4}$ Halin used the German term 'schwach m-fach kettenförmig'.
} 
graph $H_{i j}$ is finite. (By abuse of notation we do not distinguish the additional vertices $a$ and $b$ in different graphs $H_{i j}$.)

Suppose that, for a fixed value of $i$, there are always for $j$ big enough at least $k$ distinct $a-b$ paths in $H_{i j}$ such that any two of them interesect only in the vertices $a$ and $b$. Then one can use the same argument as in the proof of König's Infinity Lemma to show that then $G$ contains a family of $k$ pairwise disjoint rays. Because $G$ does not contain a family of $m+1$ pairwise disjoint rays there are for each $i$ a number $j_{i}$ such that for every $j \geq j_{i}$ there are at most $m$ disjoint $a-b$ paths in $H_{i j_{i}}$. Since $a$ and $b$ are not adjacent in $H_{i j_{i}}$ then the Menger Theorem says that minimum number of a vertices in an $a-b$ separator is equal to the maximal number of $a-b$ paths such that any two of the paths have no inner vertices in common. Whence there is in $H_{i j_{i}} \backslash\{a, b\}$ a set $T$ and $a-b$ separator with precisely $m$ vertices. This set is also an separator in $G$ and every ray in $G$ that has its initial vertex in $B_{i}$ must intersect $T$. From this information we can easily construct our sequence of separators $T_{1}, T_{2}, \ldots$.

We can also clearly assume that if $i_{j}$ is the smallest number such that $T_{j}$ is in $B_{i_{j}}$ then $T_{k} \cap B_{i_{j}}=\emptyset$ for all $k>j$.

Corollary A.2. Let $G$ be a connected locally finite graph. Suppose $\omega$ is an end of $G$ and $\omega$ has finite vertex degree $m$. Then there is a sequence $T_{1}, T_{2}, \ldots$ of separators each containing precisely $m$ vertices such that if $C_{i}$ denotes the component of $G-T_{i}$ that $\omega$ belongs to then $C_{1} \supseteq C_{2} \supseteq \ldots$ and $\bigcap_{i=1}^{\infty} C_{i}=\emptyset$.

Proof. We use exactly the same argument as above except that when we construct the $H_{i j}$ we only put in edges from $b$ to those vertices in $E_{j}$ that are in the boundary of the component of $G \backslash B_{j}$ that $\omega$ lies in.

Proof of Lemma 2.5. The first part of the Lemma about the existence of a family of $k$ pairwise disjoint rays in $\omega$ with their initial vertices in $A \cap B$ follows directly from the above.

For the second part, the only thing we need to show is that there cannot exist a separation $(C, D)$ of order $<k$ such that $A \subseteq C$ and $\omega$ lies in $D$. Such a separation cannot exist because the $k$ pairwise disjoint rays that have their initial vertices in $A \cap B$ and belong to $\omega$ would all have to pass through $C \cap D$.

Theorem A.3. (12, Satz 2]) Let $G$ be a graph with the property that it contains a family of $m$ pairwise disjoint rays but no family of $m+1$ pairwise disjoint rays. Let $X$ denote the set of vertices in $G$ that dominate some ray. Then the set $X$ is finite and the graph $G-X$ has a ray decomposition of adhesion $m$.

Proof. Let $R_{1}, \ldots, R_{m}$ denote a family of pairwise disjoint rays. Set $R=R_{1} \cup$ $\cdots \cup R_{m}$.

Any ray in $G$ must intersect the set $R$ in infinitely many vertices and thus intersects one of the rays $R_{1}, \ldots, R_{m}$ in infinitely many vertices. From this we conclude that every ray in $G$ is in the same end as one of the rays $R_{1}, \ldots, R_{m}$. Thus a vertex that dominates some ray in $G$ must dominate one of the rays $R_{1}, \ldots, R_{m}$.

In Lemma 2.3 we have already shown that the set of vertices dominating an end of finite vertex degree is finite. Note also that if a vertex in $R$ is in infinitely many distinct sets of the type $\partial C$ where $C$ is a component of $G \backslash R$ then $x$ would be a dominating vertex of some ray $R_{i}$. Thus there can only be finitely many vertices in $R$ with this property. 
We will now show that $G-X$ has a ray decomposition of adhesion $m$. To simplify the notation we will in the rest of the proof assume that $X$ is empty.

Assume now that there is a component $C$ of $G-R$ such that $\partial C$ is infinite. Take a spanning tree of $C$ and then adjoin the vertices in $\partial C$ to this tree using edges in $G$. Now we have a tree with infinitely many leafs. It is now apparent that either the tree contains a ray that does not intersect $R$ or there is a vertex in $C$ that dominates a ray in $G$. Both possibilities are contrary to our assumptions and we can conclude that $\partial C$ is finite for every component $C$ of $G \backslash R$.

For every set $S$ in $R$ of such that $S=\partial C$ for some component $C$ in $G \backslash R$ we find a locally finite connected subgraph $C_{S}$ of $C \cup S$ containing $S$. The graph $G^{\prime}$ that is the union of $R$ and all the subgraphs $C_{S}$ is a locally finite graph. The original graph $G$ has a ray decomposition of adhesion $m$ if and only if $G^{\prime}$ has a ray decomposition of adhesion $m$.

At this point we apply Theorem A.1. From Theorem A.1 we have the sequence $T_{2}, T_{3}, \ldots$ of separators. We choose $T_{2}$ such that all the rays $R_{1}, \ldots, R_{m}$ intersect $T_{2}$. We start by defining $G_{i}$ for $i \geq 2$ as the union of $T_{i}$ and all those components of $G-T_{i}$ that contain the tail of some ray $R_{i}$. Finally, set $G_{1}=G \backslash\left(G_{2} \backslash T_{2}\right)$. Note that none of the subgraphs $G_{i}$ can contain a ray and our family of rays provides a family of $m$ pairwise disjoint $T_{i}-T_{i+1}$ paths. Now we have shown that $G$ has a ray decomposition of adhesion $m$.

Finally, we are now ready to show how Halin's result above implies Theorem 2.6 that concerns $\omega$-relevant separations.

Proof of Theorem 2.6. We continue with the notation in the proof of Theorem A.3. Recall that there are infinitely many pairwise disjoint paths connecting a ray $R_{i}$ to a ray $R_{j}$. Thus we may assume that the initial vertices of the rays $R_{1}, \ldots R_{k}$ all belong to the same component of $G-T_{2}$. We set $A_{n}$ as the union of the component of $G-T_{n+1}$ that contains these initial vertices with $T_{n+1}$. Then set $B_{n}=\left(G \backslash A_{n}\right) \cup T_{n+1}$. Now it is trivial to check that the sequence $\left(A_{n}, B_{n}\right)$ of separations satisfies the conditions.

\section{B Appendix}

Proof of Lemma 4.1. Let $\sigma$ be an automorphism of $T$. In cite [24, Proposition 3.2] Tits proved that there are three types of automorphisms of a tree: (i) those that fix some vertex, (ii) those that fix no vertex but leave an edge invariant and (iii) those that leave some double-ray $\ldots, v_{-1}, v_{0}, v_{1}, v_{2}, \ldots$ invariant and act as non-trivial translations on that double-ray. (Similar results were proved independently by Halin in 13.) Since $T$ is one-ended it contains no double-ray and thus (iii) is impossible. Suppose now that $\sigma$ fixes no vertex in $T$ but leaves the edge $e$ invariant. The end of $T$ lies in one of the components of $T-e$ and $\sigma$ swaps the two components of $T-e$. This is impossible, because $T$ has only one end and this end must belong to one of the components of $T-e$. Hence $\sigma$ must fix some vertex $v$. There is a unique ray $R^{\prime}$ in $T$ with $v$ as an initial vertex and this ray is fixed pointwise by $\sigma$. The two rays $R$ and $R^{\prime}$ intersect in a ray that is a tail of $R$ and this tail of $R$ is fixed pointwise by $\sigma$. 


\section{Appendix}

In this Appendix we prove Lemma 4.2 which is a slightly sharpened version of Lemma 3 from Halin's paper [17. The change is that 'uncountable' in Halin's results is replaced by 'at least $2^{\aleph_{0}}$ elements'.

First there is an auxilliary result that corresponds to Lemma 2 in [17.

Lemma C.1. Let $G$ be a connected graph and $\Gamma=\operatorname{Aut}(G)$. Suppose $D$ is a subset of the vertex set of $G$. Let $\left\{C_{i}\right\}_{i \in I}$ denote the family of components of $G-D$. Define $G_{i}$ as the subgraph spanned by $C_{i} \cup \partial C_{i}$. Set $\Gamma_{i}=\operatorname{Aut}\left(G_{i}\right)_{\left(\partial C_{i}\right)}$. Suppose that $\Gamma_{i}$ is either finite or has at least $2^{\aleph_{0}}$ elements for all $i$. Then $\Gamma_{(D)}$ is either finite or has at least $2^{\aleph_{0}}$ elements.

Proof. If one of the groups $\gamma_{i}$ has at least $2^{\aleph_{0}}$ elements then there is nothing more to do. So, we assume that all these groups are finite.

Now there are two situations where it is possible that $\Gamma_{(D)}$ is infinite. The first is when infinitely many of the groups $\Gamma_{i}$ are non-trivial. For any family $\left\{\sigma_{i}\right\}_{i \in I}$ such that $\sigma_{i} \in \Gamma_{i}$ we can find an automorphism $\sigma \in \Gamma_{\left(G \backslash C_{i}\right)} \subseteq \Gamma_{(D)}$ such that the restriction to $C_{i}$ equals $\sigma_{i}$ for all $i$. If infinitely many of the groups $\Gamma_{C_{i}}$ are nontrivial, then there are at least $2^{\aleph_{0}}$ such families $\left\{\sigma_{i}\right\}_{i \in I}$ and $\Gamma_{(D)}$ must have at least $2^{\aleph_{0}}$ elements.

We say that two components $C_{i}$ and $C_{j}$ are equivalent if $\partial C_{i}=\partial C_{j}$ and there is an isomorphism $\varphi_{i j}$ from the subgraph $G_{i}$ to the subgraph $G_{j}$ fixing every vertex in $\partial C_{i}=\partial C_{j}$. Clearly there is an automorpism $\sigma_{i j}$ of $G$ that fixes every vertex that is neither in $C_{i}$ nor $C_{j}$ such that $\sigma_{i j}(v)=\varphi_{i j}(v)$ for $v \in C_{i}$ and $\sigma_{i j}(v)=\varphi_{i j}^{-1}(v)$ for $v \in C_{j}$. If there are infinitely many disjoint ordered pairs of equivalent components we can for any subset of these pairs find an automorphism $\sigma \in \Gamma_{(D)}$ such that if $\left(C_{i}, C_{j}\right)$ is in our subset then the restriction of $\sigma$ to $C_{i} \cup C_{j}$ is equal to the restriction of $\sigma_{i j}$. There are at least $2^{\aleph_{0}}$ such sets and thus $\Gamma_{(D)}$ has at least $2^{\aleph_{0}}$ elements.

If neither of the two cases above occurs then $\Gamma_{(D)}$ is clearly finite.

Proof of Lemma 4.2. Following Schmidt 22] (see also Halin's paper [16, Section $3]$ ) we define, using induction, for each ordinal $\lambda$ a class of graphs $A(\lambda)$. The class $A(0)$ is the class of finite graphs. Suppose $\lambda>0$ and $A(\mu)$ has already been defined for all $\mu<\lambda$. A graph $G$ is in the class $A(\lambda)$ if and only if it contains a finite set $F$ of vertices such that each component of $G-F$ is in $A(\mu)$ for some $\mu<\lambda$. It is shown in the papers referred to above that if $G$ belongs to $A(\lambda)$ for some ordinal $\lambda$ then $G$ is rayless and, conversely, every rayless graph belongs to $A(\lambda)$ for some ordinal $\lambda$. For a rayless graph $G$ we define $o(G)$ as the smallest ordinal $\lambda$ such that $G$ is in $A(\lambda)$.

The Lemma is proved by induction over $o(G)$. If $o(G)=0$ then the graph $G$ is finite and the automorphism group is also finite.

Assume that the result is true for all rayless graphs $H$ such that $o(H)<o(G)$. Find a finite set $F$ of vertices such that each of the components of $G-F$ has a smaller order than $G$. Denote the family of components of $G-F$ with $\left\{C_{i}\right\}_{i \in I}$. Denote with $G_{i}$ the subgraph induced by $C_{i} \cup \partial C_{i}$. By induction hypothesis the pointwise stabiliser of $\partial C_{i}$ in $\operatorname{Aut}\left(G_{i}\right)$ is either finite or has at least $2^{\aleph_{0}}$ elements. Lemma C.1 above implies that $\operatorname{Aut}(G)_{(D)}$ is either finite or has at least $2^{\aleph_{0}}$ elements. 


\section{References}

[1] D. Boutin and W. Imrich. The cost of distinguishing graphs. In T. Ceccherini-Silberstein, M. Salvatori, and E. Sava-Huss, editors, Groups, Graphs and Random Walks, London Mathematical Society Lecture Note Series. Cambridge University Press, publication planned for April 2017.

[2] P. J. Cameron. Oligomorphic permutation groups. Cambridge: Cambridge University Press, 1990.

[3] J. Carmesin, R. Diestel, M. Hamann, and F. Hundertmark. k-blocks: a connectivity invariant for graphs. SIAM J. Discrete Math., 28(4):18761891, 2014.

[4] J. Carmesin, R. Diestel, F. Hundertmark, and M. Stein. Connectivity and tree structure in finite graphs. Combinatorica, 34(1):11-45, 2014.

[5] W. Dicks and M. Dunwoody. Groups acting on graphs. Cambridge etc.: Cambridge University Press, 1989.

[6] R. Diestel. Graph theory. 4th ed. Berlin: Springer, 4th ed. edition, 2010.

[7] R. Diestel, H. A. Jung, and R. G. Möller. On vertex transitive graphs of infinite degree. Arch. Math., 60(6):591-600, 1993.

[8] M. J. Dunwoody. Structure trees, networks and almost invariant sets. In Groups, graphs and random walks. Selected papers of the workshop, Cortona, Italy, June 2-6, 2014 on the occasion of the 60th birthday of Wolfgang Woess, pages 137-175. Cambridge: Cambridge University Press, 2017.

[9] M. J. Dunwoody and B. Krön. Vertex cuts. J. Graph Theory, 80(2):136171, 2015.

[10] R. Halin. Über trennende Eckenmengen in Graphen und den Mengerschen Satz. Math. Ann., 157:34-41, 1964.

[11] R. Halin. Über unendliche Wege in Graphen. Math. Ann., 157:125-137, 1964.

[12] R. Halin. Über die Maximalzahl fremder unendlicher Wege in Graphen. Math. Nachr., 30:63-85, 1965.

[13] R. Halin. Automorphisms and endomorphisms of infinite locally finite graphs. Abh. Math. Semin. Univ. Hamb., 39:251-283, 1973.

[14] R. Halin. Lattices of cuts in graphs. Abh. Math. Semin. Univ. Hamb., 61:217-230, 1991.

[15] R. Halin. Some finiteness results concerning separation in graphs. Discrete Math., 101(1-3):97-106, 1992.

[16] R. Halin. The structure of rayless graphs. Abh. Math. Semin. Univ. Hamb., $68: 225-253,1998$.

[17] R. Halin. A note on graphs with countable automorphism group. Abh. Math. Semin. Univ. Hamb., 70:259-264, 2000. 
[18] M. Hamann. End-transitive graphs. Isr. J. Math., 189:437-459, 2012.

[19] M. Hamann and F. Hundertmark. The classification of connectedhomogeneous digraphs with more than one end. Trans. Am. Math. Soc., 365(1):531-553, 2013.

[20] H. A. Jung. A note on fragments of infinite graphs. Combinatorica, 1:285$288,1981$.

[21] B. Krön and R. G. Möller. Quasi-isometries between graphs and trees. J. Comb. Theory, Ser. B, 98(5):994-1013, 2008.

[22] R. Schmidt. Ein Ordnungsbegriff für Graphen ohne unendliche Wege mit einer Anwendung auf n-fach zusammenhaengende Graphen. Arch. Math., 40:283-288, 1983.

[23] C. Thomassen. The Hadwiger number of infinite vertex-transitive graphs. Combinatorica, 12(4):481-491, 1992.

[24] J. Tits. Sur le groupe des automorphismes d'un arbre. Essays Topol. Relat. Top., Mém. dédiés à Georges de Rham, 188-211 (1970)., 1970. 